\title{
Efficacy of Etanercept in the Treatment of Stevens-Johnson Syndrome and Toxic Epidermal Necrolysis
}

\author{
Sean David Dreyer, MD; Juan Torres, MD; Marie Stoddard, MD; Erica Leavitt, MD; \\ Adam Sutton, MD, MBA; Maria Aleshin, MD; Ashley Crew, MD; Scott Worswick, MD
}

\section{PRACTICE POINTS}

- Stevens-Johnson syndrome (SJS) and toxic epidermal necrolysis (TEN) are life-threatening dermatologic emergencies without a universally accepted treatment.

- Results of this study support the use of single-dose subcutaneous etanercept $50 \mathrm{mg}$ as a potentially lifesaving therapy for patients with SJS/TEN.

It has been suggested that the use of etanercept for treatment of Stevens-Johnson syndrome (SJS) and toxic epidermal necrolysis (TEN) might provide improved mortality benefit and decreased skin healing times. This retrospective study compared the use of singledose subcutaneous etanercept to intravenous immunoglobulin (IVIG) and supportive care alone. Thirteen patients were treated with a single dose $(50 \mathrm{mg})$ of subcutaneous etanercept. Results of this study support the use of etanercept as a potentially beneficial agent in the treatment of SJS/TEN.

Cutis. 2021;107:E22-E28.

$\longrightarrow$ egarded as dermatologic emergencies, StevensJohnson syndrome (SJS) and toxic epidermal necrolysis (TEN) represent a spectrum of blistering skin diseases that have a high mortality rate. Because of a misguided immune response to medications or infections, $\mathrm{CD}^{+} \mathrm{T}$ lymphocytes release proinflammatory cytokines, giving rise to the extensive epidermal destruction seen in SJS and TEN. The exact pathogenesis of SJS and TEN is still poorly defined, but studies have proposed that $\mathrm{T}$ cells mediate keratinocyte (KC) apoptosis through perforin and granzyme release and activation of the Fas/Fas ligand (FasL). Functioning as a transmembrane death receptor in the tumor necrosis factor (TNF) superfamily, Fas (CD95) activates Fas-associated death domain protein, caspases, and nucleases, resulting in organized cell destruction. Likewise, perforin and granzymes also have been shown to play a similar role in apoptosis via activation of caspases. ${ }^{1}$

Evidence for the role of TNF- $\alpha$ in SJS and TEN has been supported by findings of elevated levels of TNF- $\alpha$ within the blister fluid, serum, and $\mathrm{KC}$ cell surface. Additionally, TNF- $\alpha$ has been shown to upregulate inducible nitric oxide synthase in KCs, causing an accumulation of nitric oxide and subsequent FasL-mediated cell death. ${ }^{1-3}$ Notably, studies have demonstrated a relative lack of lymphocytes in the tissue of TEN patients despite the extensive destruction that is observed, thus emphasizing the importance of amplification and cell signaling via inflammatory mediators such as TNF- $\alpha .{ }^{1}$ In this proposed model, $\mathrm{T}$ cells release IFN- $\gamma$, causing $\mathrm{KC}$ s to release TNF- $\alpha$ that subsequently promotes the upregulation of the aforementioned FasL. ${ }^{1}$ Tumor necrosis factor $\alpha$ also may promote increased MHC class I complex deposition on KC surfaces that may play a role in perforin and granzyme-mediated apoptosis of KCs. ${ }^{1}$

Drs. Dreyer, Torres, and Leavitt are from the Division of Dermatology, David Geffen School of Medicine, University of California, Los Angeles. Dr. Stoddard is from the Department of Dermatology, University of Michigan, Ann Arbor. Dr. Sutton is from the Department of Dermatology, University of Nebraska, Lincoln. Dr. Aleshin is from the Department of Dermatology, Stanford University, California. Drs. Crew and Worswick are from the Department of Dermatology, Keck School of Medicine, University of Southern California, Los Angeles.

The authors report no conflict of interest.

Correspondence: Sean David Dreyer, MD, David Geffen School of Medicine, 10833 Le Conte Ave, Los Angeles, CA 90095 (seandreyer1@gmail.com). doi: $10.12788 /$ cutis. 0288 
There is still debate on the standard of care for the treatment of SJS and TEN, attributed to the absence of randomized controlled trials and the rarity of the disease as well as the numerous conflicting studies evaluating potential treatments. ${ }^{1,4}$ Despite conflicting data to support their use, supportive care and intravenous immunoglobulin (IVIG) continue to be common treatments for SJS and TEN in hospitals worldwide. Elucidation of the role of TNF- $\alpha$ has prompted the use of infliximab and etanercept. In a case series of Italian patients with TEN (average SCORTEN, 3.6) treated with the TNF- $\alpha$ antagonist etanercept, no mortality was observed, which was well below the calculated expected mortality of $46.9 \%{ }^{2}$ Our retrospective study compared the use of a TNF antagonist to other therapies in the treatment of SJS/ TEN. Our data suggest that etanercept is a lifesaving and disease-modifying therapy.

\section{Methods}

Twenty-two patients with SJS/TEN were included in this analysis. This included all patients who carried a clinical diagnosis of SJS/TEN with a confirmatory biopsy at our 2 university centers-University of California, Los Angeles, and Keck-LA County-Norris Hospital at the University of Southern California, Los Angeles-from 2013 to 2016. The diagnosis was rendered when a clinical diagnosis of SJS/TEN was given by a dermatologist and a confirmatory biopsy was performed. Every patient given a diagnosis of SJS/TEN at either university system from 2015 onward received an injection of etanercept given the positive results reported by Paradisi et al. ${ }^{2}$

The 9 patients who presented from 2013 to 2014 to our 2 hospital systems and were given a diagnosis of SJS/ TEN received either IVIG or supportive care alone and had an average body surface area (BSA) affected of $23 \%$. The 13 patients who presented from 2015 to 2016 were treated with etanercept in the form of a $50-\mathrm{mg}$ subcutaneous injection given once to the right upper arm. Of this group, 4 patients received dual therapy with both IVIG and etanercept. In the etanercept-treated group (etanercept alone and etanercept plus IVIG), the average BSA affected was $30 \%$. At the time of preliminary diagnosis, all patient medications were evaluated for a possible temporal relationship to the onset of rash and were discontinued if felt to be causative. The causative agent and treatment course for each patient is summarized in Table 1.

Patients were monitored daily in the hospital for improvement, and time to re-epithelialization was measured. Re-epithelialization was defined as progressive healing with residual lesions (erosions, ulcers, or bullae) covering no more than 5\% BSA and was contingent on the patient having no new lesions within 24 hours. $^{5}$ SCORe of Toxic Epidermal Necrosis (SCORTEN), a validated severity-of-illness score, ${ }^{6}$ was calculated by giving 1 point for each of the following criteria at the time of diagnosis: age $\geq 40$ years, concurrent malignancy, heart rate $\geq 120$ beats/min, serum blood urea nitrogen $>27 \mathrm{mg} / \mathrm{dL}$, serum bicarbonate $<20 \mathrm{mEq} / \mathrm{L}$, serum glucose $>250 \mathrm{mg} / \mathrm{dL}$, and detached or compromised BSA $>10 \%$. The total SCORTEN was correlated with the following risk of mortality as supported by prior validation studies: SCORTEN of 0 to 1, 3.2\%; SCORTEN of 2, 12.1\%; SCORTEN of 3, 35.3\%; SCORTEN of 4 , $58.3 \%$; SCORTEN of $\geq 5,>90 \%$.

\section{Results}

A total of 13 patients received etanercept. The mean SCORTEN was 2.2. The observed mortality was $0 \%$, which was markedly lower than the predicted mortality of $24.3 \%$ (as determined by linear interpolation). Of this cohort, 9 patients received etanercept alone (mean SCORTEN of 2.1, predicted mortality of $22.9 \%$ ), whereas 4 patients received a combination of etanercept and IVIG (mean SCORTEN of 2.3, predicted mortality of 27.2\%).

The 4 patients who received both etanercept and IVIG received dual therapy for varying reasons. In patient 2 (Table 1), the perceived severity of this case ultimately led to the decision to start IVIG in addition to etanercept, resulting in rapid recovery and discharge after only 1 week of hospitalization. Intravenous immunoglobulin also was given in patient 3 (SCORTEN of 4) and patient 6 (SCORTEN of 2) for progression of disease despite administration of etanercept, with subsequent cessation of progression after the addition of the second agent (IVIG). Patient 12 might have done well on etanercept monotherapy but was administered IVIG as a precautionary measure because of hospital treatment algorithms.

Nine patients did not receive etanercept. Of this group, 5 received IVIG and 4 were managed with supportive care alone. The average SCORTEN for this group was 2.4, only slightly higher than the group that received etanercept (Table 2). The mortality rate in this group was 33\%, which was higher than the predicted mortality of $28.1 \%$.

Re-epithelialization data were available for 8 patients who received etanercept. The average time to re-epithelialization for these patients was 8.9 days and ranged from 3 to 19 days. Of these patients, 2 received both IVIG and etanercept, with an average time to re-epithelialization of 13 days. For the 6 patients who received etanercept alone, the average time to reepithelialization was 7.5 days. Re-epithelialization data were not available for any of the patients who received only IVIG or supportive care but to our recollection ranged from 14 to 21 days.

The clinical course of the 13 patients after the administration of a single dose of etanercept was remarkable, as there was complete absence of mortality and an increase in speed of recovery in most patients receiving this intervention (time to re-epithelialization, 3-19 days). We also observed another interesting trend from our patients treated with etanercept, which was the suggestion that treatment with etanercept may be less effective if IVIG and/or steroids are given prior to etanercept; 


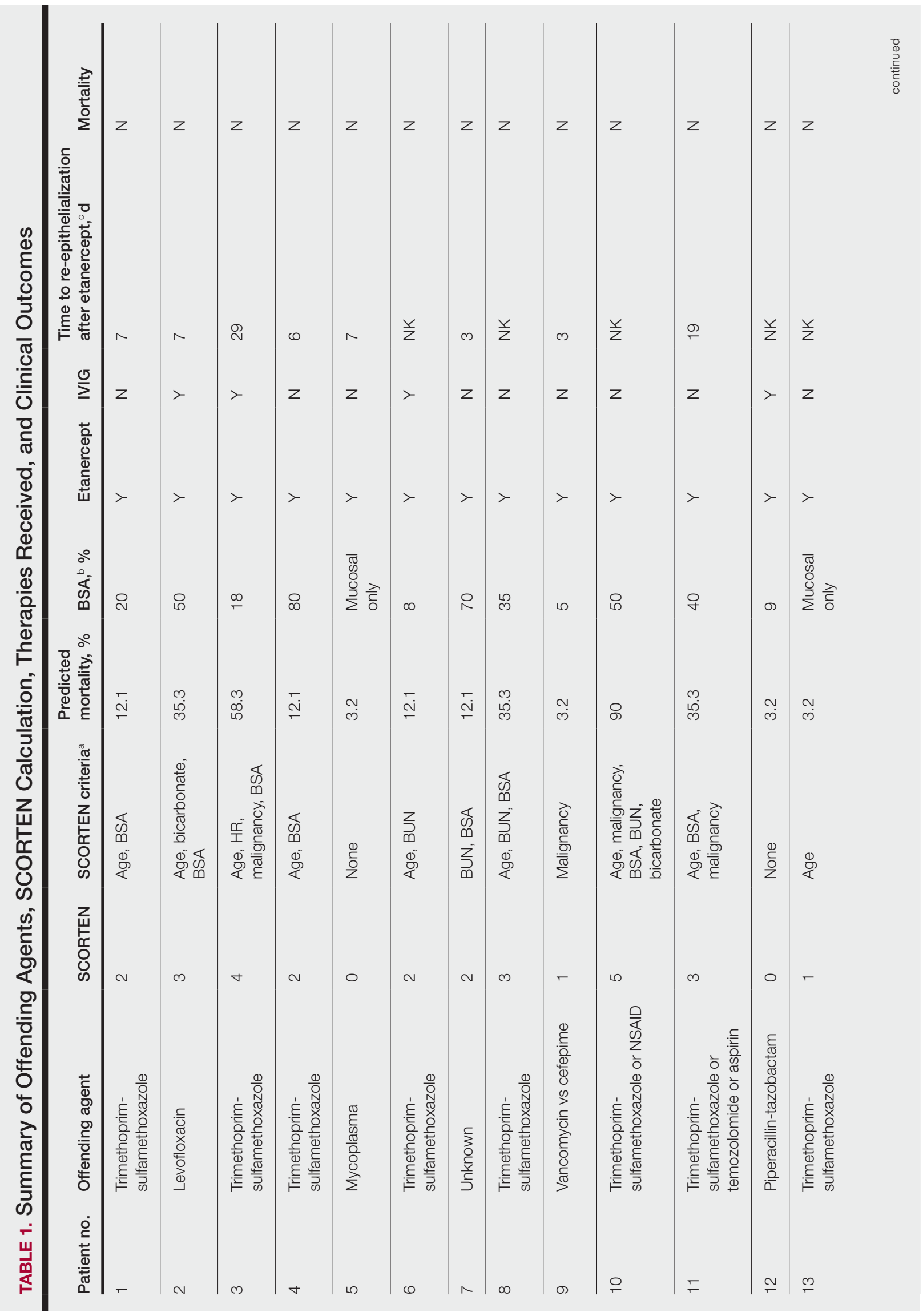




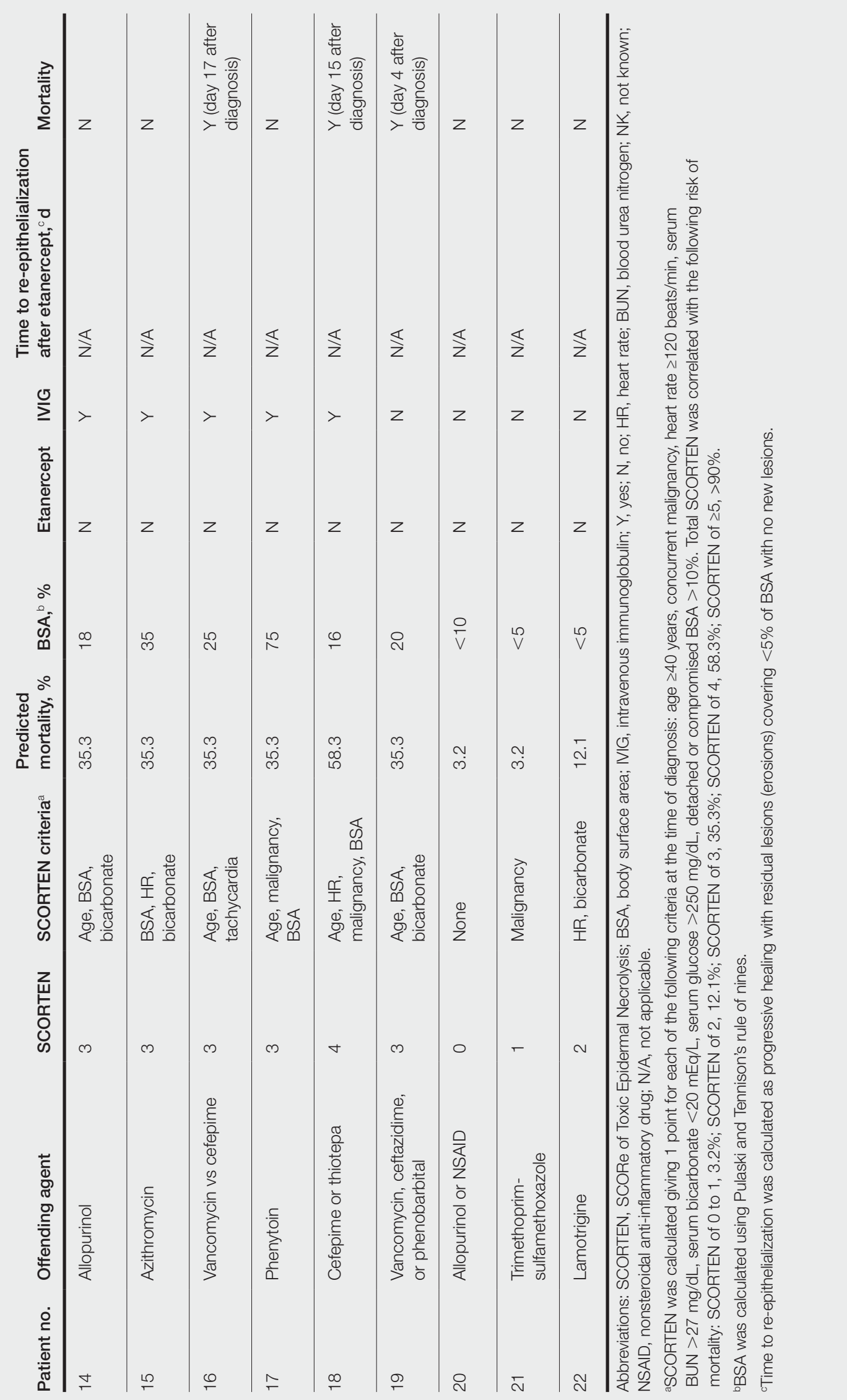


TABLE 2. Observed Mortality vs Predicted Mortality ${ }^{a}$ Among Treatment Groups

\begin{tabular}{lllll} 
& & No. of & & \\
Treatment received & Mean SCORTEN & patients & Observed mortality, $\mathbf{n}(\%)$ & Predicted mortality, \% \\
\hline Etanercept + /- IVIG & 2.2 & 13 & $0 / 13(0)$ & 24.3 \\
\hline Etanercept + IVIG & 2.3 & 4 & $0 / 4(0)$ & 27.2 \\
\hline Etanercept alone & 2.1 & 9 & $0 / 9(0)$ & 22.9 \\
\hline No etanercept (IVIG or supportive care) & 2.4 & 9 & $3 / 9(33)$ & 28.1 \\
\hline
\end{tabular}

Abbreviations: SCORTEN, SCORe of Toxic Epidermal Necrolysis; IVIG, intravenous immunoglobulin.

aBased on calculated SCORTEN.

likewise, treatment is more effective when etanercept is given quickly. For patients 1, 4, 5, 7, 9, and 11 (as shown in Table 1), no prior IVIG therapy or other immunosuppressive therapy had been given before etanercept was administered. In these 6 patients, the average time to re-epithelialization after etanercept administration was 7.5 days; average time to re-epithelialization, unfortunately, is not available for the patients who were not treated with etanercept. In addition, as shown in the Figure, it was noted in some patients that the depth of denudation was markedly more superficial than what would typically be clinically observed with TEN after administration of other immunomodulatory therapies such as IVIG or prednisone or with supportive care alone. In these 2 patients with superficial desquamationpatients 7 and 9-etanercept notably was given within 6 hours of onset of skin pain.

\section{Comment}

There is no definitive gold standard treatment of SJS, SJS/TEN overlap, or TEN. However, generally agreed upon management includes immediate discontinuation of the offending medication and supportive therapy with aggressive electrolyte replacement and wound care. Management in a burn unit or intensive care unit is recommended in severe cases. Contention over the efficacy of various medications in the treatment of SJS and TEN continues and largely is due to the rarity of SJS and TEN; studies are small and almost all lack randomization. Therapies that have been used include high-dose steroids, IVIG, plasmapheresis, cyclophosphamide, cyclosporine A, and TNF inhibitors (eg, etanercept, infliximab). ${ }^{1}$

Evidence for the use of anti-TNF- $\alpha$ antibodies has been limited thus far, with most of the literature focusing on infliximab and etanercept. Adalimumab, a fully humanized clonal antibody, has no reported cases in the dermatologic literature for use in patients with SJS/TEN. Two case reports of adalimumab paradoxically causing SJS have been documented. In both cases, adalimumab was stopped and patients responded to intravenous
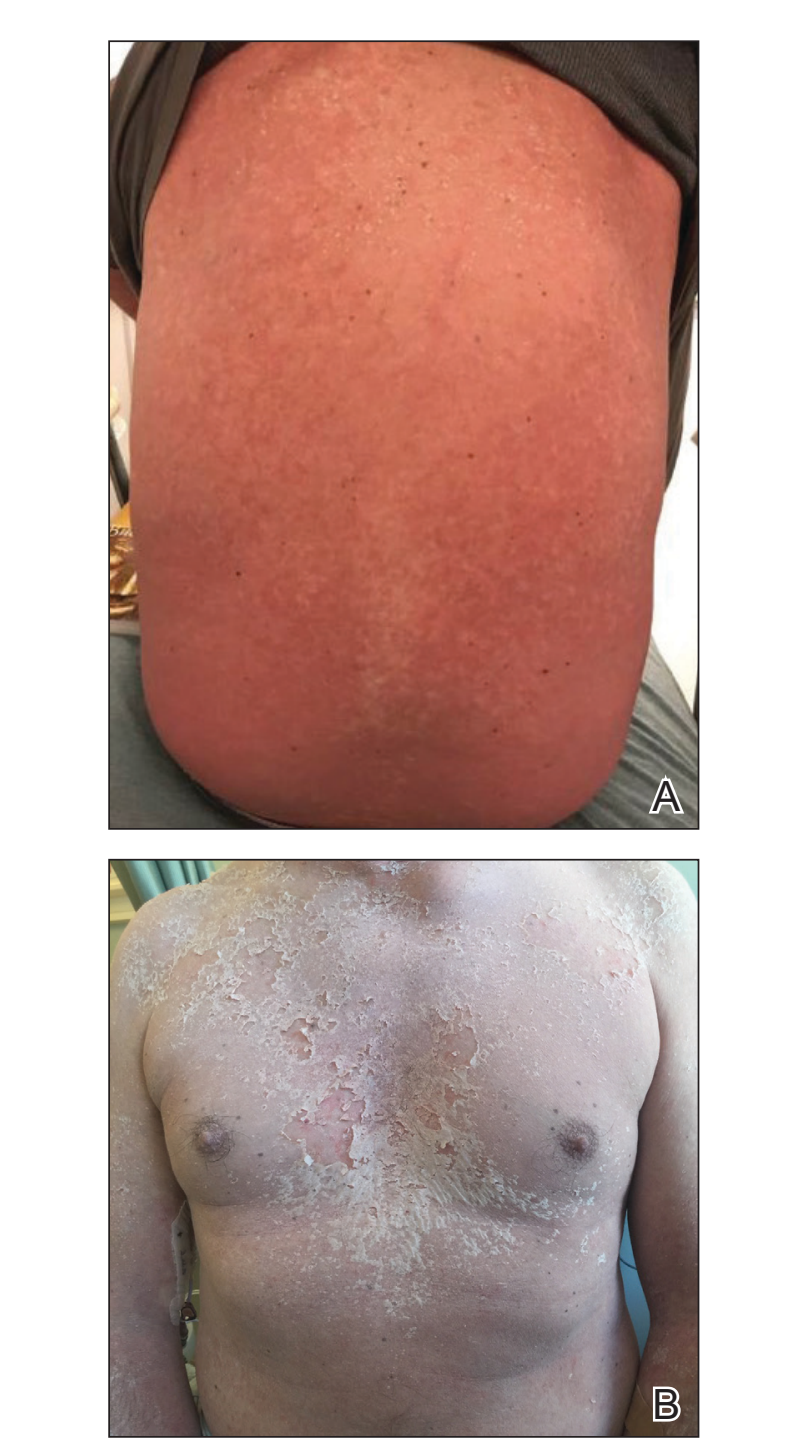

A, Dusky erythema covering $80 \%$ of the patient's body surface area, suggestive of incipient full-thickness epidermal necrosis, 1 hour prior to etanercept administration (patient 4). B, Superficial desquamation mimicking sunburn 7 days after etanercept administration. 
corticosteroids and infliximab. ${ }^{7,8}$ Similarly, thalidomide has not proven to be a promising anti-TNF- $\alpha$ agent for the treatment of SJS/TEN. In the only attempted randomized controlled trial for SJS and TEN, thalidomide appeared to increase mortality, eventuating in this trial being terminated prior to the planned end date. ${ }^{9}$

Infliximab and etanercept have several case reports and a few case series highlighting potentially efficacious application of TNF- $\alpha$ inhibitors for the treatment of SJS/ TEN. ${ }^{10-13}$ In 2002, Fischer et $\mathrm{al}^{10}$ reported the first case of TEN treated successfully with a single dose of infliximab $5 \mathrm{mg} / \mathrm{kg}$. Kreft et $\mathrm{al}^{14}$ reported on etoricoxib-induced TEN that was treated with infliximab $5 \mathrm{mg} / \mathrm{kg}$, which led to re-epithelialization within 5 weeks (notably a 5 -week reepithelialization time is not necessarily an improvement).

In 2005, Hunger et $\mathrm{al}^{3}$ demonstrated TNF- $\alpha$ 's release by KCs in the epidermis and by inflammatory cells in the dermis of a TEN patient. Twenty-four hours after the administration of infliximab $5 \mathrm{mg} / \mathrm{kg}$ in these patients, TNF- $\alpha$ was found to be below normal and epidermal detachment ceased. ${ }^{3}$ Wojtkietwicz et al $^{13}$ demonstrated benefit following an infusion of infliximab $5 \mathrm{mg} / \mathrm{kg}$ in a patient whose disease continued to progress despite treatment with dexamethasone and $1.8 \mathrm{~g} / \mathrm{kg}$ of IVIG.

Then 2 subsequent case series added further support for the efficacy of infliximab in the treatment of TEN. Patmanidis et $\mathrm{al}^{15}$ and Gaitanis et $\mathrm{al}^{16}$ reported similar results in 4 patients, each treated with infliximab $5 \mathrm{mg} / \mathrm{kg}$ immediately followed by initiation of high-dose IVIG ( $2 \mathrm{~g} / \mathrm{kg}$ over 5 days). Zárate-Correa et a ${ }^{17}$ reported a $0 \%$ mortality rate and near-complete re-epithelialization after 5 to 14 days in 4 patients treated with a single 300-mg dose of infliximab.

However, the success of infliximab in the treatment of TEN has been countered by the pilot study by Paquet et $\mathrm{al}_{1}{ }^{18}$ which compared the efficacy of $150 \mathrm{mg} / \mathrm{kg}$ of $\mathrm{N}$-acetylcysteine alone vs adding infliximab $5 \mathrm{mg} / \mathrm{kg}$ to treat 10 TEN patients. The study demonstrated no benefit at 48 hours in the group given infliximab, the time frame in which prior case reports touting infliximab's benefit claimed the benefit was observed. Similarly, there was no effect on mortality for either treatment modality as assessed by illness auxiliary score. ${ }^{18}$

Evidence in support of the use of etanercept in the treatment of SJS/TEN is mounting, and some centers have begun to use it as the first-choice therapy for SJS/TEN. The first case was reported by Famularo et al, ${ }^{19}$ in which a patient with TEN was given 2 doses of etanercept $25 \mathrm{mg}$ after failure to improve with prednisolone $1 \mathrm{mg} / \mathrm{kg}$. The patient showed near-complete and rapid re-epithelization in 6 days before death due to disseminated intravascular coagulation 10 days after admission. ${ }^{19}$ Gubinelli et $\mathrm{al}^{20}$ and Sadighha ${ }^{21}$ independently reported cases of TEN and TEN/acute generalized exanthematous pustulosis overlap treated with a total of $50 \mathrm{mg}$ of etanercept, demonstrating rapid cessation of lesion progression. Didona et $\mathrm{al}^{22}$ found similar benefit using etanercept $50 \mathrm{mg}$ to treat TEN secondary to rituximab after failure to improve with prednisone and cyclophosphamide. Treatment of TEN with etanercept in an HIV-positive patient also has been reported. Lee et $\mathrm{al}^{23}$ described a patient who was administered 50-mg and 25-mg injections on days 3 and 5 of hospitalization, respectively, with re-epithelialization occurring by day 8 . Finally, Owczarczyk-Saczonek et $\mathrm{al}^{24}$ reported a case of SJS in a patient with a 4-year history of etanercept and sulfasalazine treatment of rheumatoid arthritis; sulfasalazine was stopped, but this patient was continued on etanercept until resolution of skin and mucosal symptoms. However, it is important to consider the possibility of publication bias among these cases selected for their positive outcomes.

Perhaps the most compelling literature regarding the use of etanercept for TEN was described in a case series by Paradisi et al. ${ }^{2}$ This study included 10 patients with TEN, all of whom demonstrated complete re-epithelialization shortly after receiving etanercept $50 \mathrm{mg}$. Average SCORTEN was 3.6 with a range of 2 to 6 . Eight patients in this study had severe comorbidities and all 10 patients survived, with a time to re-epithelialization ranging from 7 to 20 days. $^{2}$ Additionally, a randomized controlled trial showed that 38 etanercept-treated patients had improved mortality $(P=.266)$ and re-epithelialization time $(P=.01)$ compared to patients treated with intravenous methylprednisolone. ${ }^{25}$

Limitations to our study are similar to other reports of SJS/TEN and included the small number of cases and lack of randomization. Additionally, we do not have data available for all patients for time between onset of disease and treatment initiation. Because of these challenges, data presented in this case series is observational only. Additionally, the patients treated with etanercept alone had a slightly lower SCORTEN compared to the group that received IVIG or supportive care alone (2.1 and 2.4 respectively). However, the etanercept-only group actually had higher involvement of epidermal detachment (33\%) compared to the non-etanercept group (23\%).

\section{Conclusion}

Although treatment with etanercept lacks the support of a randomized controlled trial, similar to all other treatments currently used for SJS and TEN, preliminary reports highlight a benefit in disease progression and improvement in time to re-epithelialization. In particular, if etanercept $50 \mathrm{mg}$ subcutaneously is given as monotherapy or is given early in the disease course (prior to other therapies being attempted and ideally within 6 hours of presentation), our data suggest an even greater trend toward improved mortality and decreased time to re-epithelialization. Additionally, our findings may suggest that in some patients, etanercept monotherapy is not an adequate intervention but the addition of IVIG may be helpful; however, the senior author (S.W.) notes anecdotally that in his experience with the patients treated at the University of California Los Angeles, the order of administration of combination therapies-etanercept followed 
by IVIG-was important in addition to the choice of therapy. These findings are promising enough to warrant a multicenter randomized controlled trial comparing the efficacy of etanercept to other more commonly used treatments for this spectrum of disease, including IVIG and/or cyclosporine. Based on the data presented in this case series, including the 13 patients who received etanercept and had a $0 \%$ mortality rate, etanercept may be viewed as a targeted therapeutic intervention for patients with SJS and TEN.

\section{REFERENCES}

1. Pereira FA, Mudgil AV, Rosmarin DM. Toxic epidermal necrolysis. J Am Acad Dermatol. 2007;56:181-200.

2. Paradisi A, Abeni D, Bergamo F, et al. Etanercept therapy for toxic epidermal necrolysis. J Am Acad Dermatol. 2014;71:278-283.

3. Hunger RE, Hunziker T, Buettiker $\mathrm{U}$, et al. Rapid resolution of toxic epidermal necrolysis with anti-TNF- $\alpha$ treatment. J Allergy Clin Immunol. 2005;116:923-924.

4. Worswick S, Cotliar J. Stevens-Johnson syndrome and toxic epidermal necrolysis: a review of treatment options. Dermatol Ther. 2011;24:207-218

5. Wallace $\mathrm{AB}$. The exposure treatment of burns. Lancet Lond Engl. 1951;1:501-504.

6. Bastuji-Garin S, Fouchard N, Bertocchi M, et al. SCORTEN: a severity-of-illness score for toxic epidermal necrolysis. J Invest Dermatol. 2000;115:149-153.

7. Mounach A, Rezqi A, Nouijai A, et al. Stevens-Johnson syndrome complicating adalimumab therapy in rheumatoid arthritis disease. Rheumatol Int. 2013;33:1351-1353.

8. Salama M, Lawrance I-C. Stevens-Johnson syndrome complicating adalimumab therapy in Crohn's disease. World J Gastroenterol. 2009;15:4449-4452.

9. Wolkenstein P, Latarjet J, Roujeau JC, et al. Randomised comparison of thalidomide versus placebo in toxic epidermal necrolysis. Lancet Lond Engl. 1998;352:1586-1589.

10. Fischer M, Fiedler E, Marsch WC, et al Antitumour necrosis factor- $\alpha$ antibodies (infliximab) in the treatment of a patient with toxic epidermal necrolysis. Br J Dermatol. 2002;146:707-709.

11. Meiss F, Helmbold P, Meykadeh N, et al. Overlap of acute generalized exanthematous pustulosis and toxic epidermal necrolysis: response to antitumour necrosis factor-alpha antibody infliximab: report of three cases. J Eur Acad Dermatol Venereol. 2007;21:717-719.

12. Al-Shouli S, Abouchala N, Bogusz MJ, et al. Toxic epidermal necrolysis associated with high intake of sildenafil and its response to infliximab. Acta Derm Venereol. 2005;85:534-535.

13. Wojtkiewicz A, Wysocki M, Fortuna J, et al. Beneficial and rapid effect of infliximab on the course of toxic epidermal necrolysis. Acta Derm Venereol. 2008;88:420-421.

14. Kreft B, Wohlrab J, Bramsiepe I, et al. Etoricoxib-induced toxic epidermal necrolysis: successful treatment with infliximab. J Dermatol. 2010;37:904-906.

15. Patmanidis K, Sidiras A, Dolianitis K, et al. Combination of infliximab and high-dose intravenous immunoglobulin for toxic epidermal necrolysis: successful treatment of an elderly patient. Case Rep Dermatol Med. 2012;2012:915314.

16. Gaitanis G, Spyridonos P, Patmanidis K, et al. Treatment of toxic epidermal necrolysis with the combination of infliximab and high-dose intravenous immunoglobulin. Dermatol Basel Switz. 2012;224:134-139.

17. Zárate-Correa LC, Carrillo-Gómez DC, Ramírez-Escobar AF, et al. Toxic epidermal necrolysis successfully treated with infliximab. J Investig Allergol Clin Immunol. 2013;23:61-63.

18. Paquet $\mathrm{P}$, Jennes $\mathrm{S}$, Rousseau AF, et al. Effect of N-acetylcysteine combined with infliximab on toxic epidermal necrolysis. a proof-ofconcept study. Burns J Int Soc Burn Inj. 2014;40:1707-1712.

19. Famularo G, Dona BD, Canzona F, et al. Etanercept for toxic epidermal necrolysis. Ann Pharmacother. 2007;41:1083-1084.

20. Gubinelli E, Canzona F, Tonanzi T, et al. Toxic epidermal necrolysis successfully treated with etanercept. J Dermatol. 2009;36:150-153.

21. Sadighha A. Etanercept in the treatment of a patient with acute generalized exanthematous pustulosis/toxic epidermal necrolysis: definition of a new model based on translational research. Int J Dermatol. 2009;48:913-914

22. Didona D, Paolino G, Garcovich S, et al. Successful use of etanercept in a case of toxic epidermal necrolysis induced by rituximab. J Eur Acad Dermatol Venereol. 2016;30:E83-E84

23. Lee $\mathrm{Y}-\mathrm{Y}, \mathrm{Ko} \mathrm{J}-\mathrm{H}$, Wei C-H, et al. Use of etanercept to treat toxic epidermal necrolysis in a human immunodeficiency virus-positive patient. Dermatol Sin. 2013;31:78-81.

24. Owczarczyk-Saczonek A, Zdanowska N, Znajewska-Pander A, et al. Stevens-Johnson syndrome in a patient with rheumatoid arthritis during long-term etanercept therapy. J Dermatol Case Rep. 2016;10:14-16.

25. Wang CW, Yang LY, Chen CB, et al. Randomized, controlled trial of TNF- $\alpha$ antagonist in CTL mediated severe cutaneous adverse reactions. J Clin Invest. 2018;128:985-996. 